\title{
Hematopoietic Cell Transplantation Cures Adenosine Deaminase 2 Deficiency: Report on 30 Patients
}

\author{
Hasan Hashem ${ }^{1} \cdot$ Giorgia Bucciol $^{2,3} \cdot$ Seza Ozen $^{4,5} \cdot$ Sule Unal ${ }^{6} \cdot$ Ikbal Ok Bozkaya $^{7}$ - Nurten Akarsu ${ }^{8}$. \\ Mervi Taskinen $^{9} \cdot$ Minna Koskenvuo $^{10}$. Janna Saarela ${ }^{11,12}$. Dimana Dimitrova ${ }^{13}$. Dennis D. Hickstein ${ }^{14}$. \\ Amy P. Hsu ${ }^{15}$. Steven M. Holland ${ }^{15} \cdot$ Robert Krance $^{16} \cdot$ Ghadir Sasa $^{16} \cdot$ Ashish R. Kumar $^{17,18}$. Ingo Müller ${ }^{19}$. \\ Monica Abreu de Sousa ${ }^{19}$. Selket Delafontaine ${ }^{2,3}$. Leen Moens ${ }^{3}$. Florian Babor ${ }^{20}$. Federica Barzaghi ${ }^{21}$. \\ Maria Pia Cicalese ${ }^{22} \cdot$ Robbert Bredius $^{23}$. Joris van Montfrans ${ }^{24}$. Valentina Baretta ${ }^{25}$. Simone Cesaro ${ }^{25}$. \\ Polina Stepensky ${ }^{26}$. Neven Benedicte ${ }^{27}$. Despina Moshous ${ }^{27}$. Guillaume Le Guenno ${ }^{28}$. David Boutboul ${ }^{29}$. \\ Jignesh Dalal ${ }^{30}$. Joel P. Brooks ${ }^{31}$. Elif Dokmeci' ${ }^{32}$. Jasmeen Dara ${ }^{33}$. Carrie L. Lucas ${ }^{31}$. Sophie Hambleton ${ }^{34}$. \\ Keith Wilson ${ }^{35}$. Stephen Jolles ${ }^{36}$. Yener Koc ${ }^{37}$. Tayfun Güngöor ${ }^{38}$. Caroline Schnider $^{39}$. Fabio Candotti ${ }^{40}$. \\ Sandra Steinmann ${ }^{41} \cdot$ Ansgar Schulz $^{41}$. Chip Chambers ${ }^{42} \cdot$ Michael Hershfield $^{43} \cdot$ Amanda Ombrello $^{44}$. \\ Jennifer A. Kanakry ${ }^{13} \cdot$ Isabelle Meyts ${ }^{2,3}$
}

Received: 8 June 2021 / Accepted: 6 July 2021 / Published online: 29 July 2021

(C) The Author(s) 2021

\begin{abstract}
Purpose Deficiency of adenosine deaminase 2 (DADA2) is an inherited inborn error of immunity, characterized by autoinflammation (recurrent fever), vasculopathy (livedo racemosa, polyarteritis nodosa, lacunar ischemic strokes, and intracranial hemorrhages), immunodeficiency, lymphoproliferation, immune cytopenias, and bone marrow failure (BMF). Tumor necrosis factor $(\mathrm{TNF}-\alpha)$ blockade is the treatment of choice for the vasculopathy, but often fails to reverse refractory cytopenia. We aimed to study the outcome of hematopoietic cell transplantation (HCT) in patients with DADA2.

Methods We conducted a retrospective study on the outcome of HCT in patients with DADA2. The primary outcome was overall survival (OS).

Results Thirty DADA2 patients from 12 countries received a total of 38 HCTs. The indications for HCT were BMF, immune cytopenia, malignancy, or immunodeficiency. Median age at HCT was 9 years (range: 2-28 years). The conditioning regimens for the final transplants were myeloablative $(n=20)$, reduced intensity $(n=8)$, or non-myeloablative $(n=2)$. Donors were HLA-matched related $(n=4)$, HLA-matched unrelated $(n=16)$, HLA-haploidentical $(n=2)$, or HLA-mismatched unrelated $(n=8)$. After a median follow-up of 2 years (range: 0.5-16 years), 2-year OS was 97\%, and 2-year GvHD-free relapse-free survival was $73 \%$. The hematological and immunological phenotypes resolved, and there were no new vascular events. Plasma ADA2 enzyme activity normalized in $16 / 17$ patients tested. Six patients required more than one HCT.

Conclusion HCT was an effective treatment for DADA2, successfully reversing the refractory cytopenia, as well as the vasculopathy and immunodeficiency.

Clinical Implications HCT is a definitive cure for DADA2 with > 95\% survival.
\end{abstract}

Keywords Hematopoietic cell transplantation · Deficiency of adenosine deaminase 2 - DADA2 - Inborn error of immunity · Bone marrow failure $\cdot$ Immunodeficiency $\cdot$ Autoinflammation

Hasan Hashem

hh.08847@khcc.jo

Isabelle Meyts

isabelle.meyts@uzleuven.be

Extended author information available on the last page of the article

\author{
Abbreviations \\ HCT Hematopoietic cell transplantation \\ DADA2 Deficiency of adenosine deaminase type 2 \\ TNF- $\alpha$ Tumor necrosis factor alpha \\ BMF Bone marrow failure \\ PAN Polyarteritis nodosa \\ GvHD Graft-versus-host disease \\ GF Graft failure
}




$\begin{array}{ll}\text { GRFS } & \text { GvHD-free relapse-free survival } \\ \text { MAC } & \text { Myeloablative conditioning } \\ \text { RIC } & \text { Reduced intensity conditioning } \\ \text { NMA } & \text { Non-myeloablative conditioning } \\ \text { PRCA } & \text { Pure red cell aplasia } \\ \text { MSD } & \text { HLA-matched sibling donor } \\ \text { MUD } & \text { HLA-matched unrelated donor } \\ \text { ATG } & \text { Anti-thymocyte globulin }\end{array}$

\section{Introduction}

In 2014, biallelic deleterious mutations in the cat eye chromosome region 1 gene (CECRI, subsequently renamed $A D A 2$ ), encoding adenosine deaminase 2 (ADA2), were reported as the cause of a monogenic inborn error of immunity disease, deficiency of ADA2 (DADA2) (OMIM \# $615,688)[1,2]$. The phenotype comprises recurrent fever and vasculopathy, ranging from livedo racemosa and polyarteritis nodosa (PAN) to intracranial vasculopathy with lacunar strokes and hemorrhages [1-3]. Cytopenias, either autoimmune or due to bone marrow failure (BMF), occur in $50 \%$ of patients and present as congenital pure red cell aplasia (PRCA), neutropenia, thrombocytopenia, or pancytopenia [4-6]. Immunodeficiency with hypogammaglobulinemia and recurrent viral and bacterial infections and malignant lymphoproliferation (T-large granular lymphocyte leukemia (T-LGL leukemia) and lymphoma) have also been described [5, 7-9]. DADA2 diagnosis is based on an absence or low levels of plasma ADA2 enzymatic activity and the demonstration of biallelic loss-of-function mutations of $A D A 2$ [10]. The pathophysiology of DADA2 remains unclear. A picture emerges where ADA2 deficiency results in skewing of macrophage differentiation towards inflammatory M1 macrophages [11], leading to endothelial instability, as shown in a zebrafish model and in endothelial cell coculture systems [1]. Recent findings have revealed an even more complex interplay between endothelial cells and monocytes and macrophages with marked ADA2 secretion by endothelial cell lines [12].

Treatment of DADA2 is challenging and case mortality is estimated to be around $8 \%$, mostly in childhood and related to vasculopathy-associated complications and infections [13-15]. None of the classical immunosuppressive drugs are an option for the long-term treatment of DADA2, because their efficacy is temporary, especially for the DADA2 related cytopenia, or because of the toxicity associated with longterm use. Anti-TNF agents, etanercept in particular, are the mainstay of treatment for the inflammatory and vasculopathy phenotypes [16]. However, anti-TNF agents do not cure the hematological phenotype and in a proportion of patients, vasculopathy persists despite anti-TNF treatment $[17,18]$. Finally, the cost of life-long TNF-inhibition is a limitation for some patients. Hematopoietic cell transplantation (HCT) has been reported to result in a rapid and sustained resolution of the systemic inflammation and hematological phenotype, with all patients from a cohort of 14 patients surviving after HCT [5, 19-21]. We report here the results of a multinational study of a cohort of 30 patients with DADA2 undergoing HCT, including the previously reported cases.

\section{Methods}

\section{Overview of the Study}

We conducted an investigator-driven retrospective international non-interventional multicenter study on HCT for DADA2. Invitations to participate were sent to the physicians allied to the DADA2 Foundation, the European Group for Blood and Marrow Transplantation (EBMT), and the European Society for Immunodeficiencies (ESID). We also invited all authors of published single case reports on HCT in DADA2 to participate in the study. Data collection began after the Second Inaugural International Conference on DADA2 hosted by the DADA2 Foundation on November 9 , 2018. The study was approved by the Ethics Committee of Leuven University Hospitals (study number S63982). The study was performed in accordance with the principles of the Declaration of Helsinki. The authors assume responsibility for the accuracy and completeness of the data and analyses and for fidelity to the study protocol.

\section{Patients}

The criteria for patient inclusion in the study were as follows: (1) genetic diagnosis of DADA2 and/or clinical findings consistent with DADA2 and plasma ADA2 activity level in the deficient range and (2) HCT performed with a follow-up time for survivors of at least 3 months after HCT. All participating physicians completed a questionnaire. All patients or their guardians gave written informed consent for data collection. Patients for whom incomplete data were obtained (indication for HCT, age at HCT, total nucleated cell dose or CD34 + stem cell dose, stem cell donor, conditioning regimen, graft-versus-host disease (GvHD) prophylaxis, time to engraftment, graft failure, conditioning for subsequent HCTs, chimerism) were excluded from the study.

\section{HCT Data}

Neutrophil engraftment was defined as the first of three consecutive days with a neutrophil count $\geq 0.5 \times 10^{9} / \mathrm{L}$ and platelet engraftment as the first of seven consecutive days with a platelet count $\geq 20 \times 10^{9} / \mathrm{L}$ without platelet transfusion in the prior 7 days. Full donor chimerism was defined as $\geq 95 \%$ 
donor cells in myeloid or whole-blood fractions. The type of test was at the discretion of the transplant center. Primary and secondary graft failures (GF) were defined according to EBMT guidelines. Second (or third) HCT was defined as the infusion of hematopoietic progenitor cell containing product, according to CIBMTR, regardless of conditioning regimen. The diagnosis and grading of acute and chronic GvHD were based on international standard criteria [22]. Transplant regimen, GvHD prophylaxis, antimicrobial prophylaxis, and pre-emptive treatment were chosen according to center preferences. Preparative regimens were classified as reduced intensity conditioning (RIC) if the dose of alkylating agents or TBI is reduced by at least 30\% from a myeloablative conditioning (MAC) approach. A total dose of treosulfan $>30 \mathrm{~g} / \mathrm{m}^{2}$ was considered MAC whether or not combined with another alkylator, whereas a total busulfan dose $<8 \mathrm{mg} / \mathrm{kg}$ and fludarabine-melphalan regimens were considered RIC.

Kaplan-Meier curves were plotted for overall survival (OS) and GvHD-free relapse-free survival (GRFS), and $p$ values were obtained for Mantel-Cox log-rank tests performed with Graph-Pad Prism Software version 9. Values of $p<0.05$ were considered statistically significant. GvHD relapse-free survival was calculated as the time from first HCT until the first occurrence of any of the following events: grades 3-4 aGvHD or moderate/severe cGvHD GF, disease relapse (poor graft function/graft failure with DADA2 disease relapse requiring repeat transplant), or death. HCTs inadvertently using affected donors were excluded. The cumulative incidence of GvHD and GF were also calculated using competing risk analysis, using R, for all HCT procedures, but excluding HCTs from affected donors.

\section{Results}

\section{Patient Characteristics and Diagnosis of DADA2}

We included 30 DADA2 patients undergoing HCT between 2000 and 2020 in this study. Four other patients were excluded due to incomplete data sets. Patients underwent HCT at 21 different centers from 12 countries in Europe and North America. Twenty of the patients have been reported before [4, 5, 7, 9, 19-21, 23-27]. Median age at disease onset was 2.25 years (range: birth to 16 years). Median age at genetic diagnosis was 12 years (range: $2-28$ years) (Table 1). DADA2 diagnosis was confirmed at the molecular level in all patients, by demonstrating the presence of biallelic pathogenic $A D A 2$ variants. Plasma ADA2 activity was assessed before HCT in 18 patients and was low in all cases. Twenty-six patients harbored known pathogenic ADA2 mutations. The R169Q variant was the most common mutation, found in 15 patients. Four patients harbored novel mutations, all with combined annotation-dependent depletion (CADD) scores above the mutation significance cutoff (MSC) for this gene; all these variants were private or had a $\mathrm{MAF}<10^{-6}[28]$, strongly suggestive of a deleterious effect. Three of these patients were tested for ADA2 enzyme activity, which was found to be low or absent (Table 2). Patient and HCT characteristics are summarized in Table S1 in the Online Supplement.

\section{Hematological Phenotype Pre-HCT}

PRCA was documented in $8 / 30$ patients, isolated neutropenia in $6 / 30$, combined RCA and neutropenia in $5 / 30$, severe aplastic anemia in $1 / 30$, severe lymphopenia in $1 / 30$, anemia and neutropenia in $2 / 30$, autoimmune hemolytic anemia (AIHA) in 2/30, and pancytopenia in 5/30 patients, at presentation. Six patients had a hematological malignancy or myelodysplasia and received HCT as part of the therapeutic approach (P4, P9, P17, P21, P26, and P27). Twenty-nine patients had received at least one immunosuppressive treatment prior to HCT including 13 patients who received at least 3 lines of immunosuppressive medications. Fourteen patients had received anti-TNF agents before HCT, without effect on cytopenias. P6 received single agent etanercept, which failed to reverse neutropenia; a combination of adalimumab, cyclosporine, and low-dose prednisone resulted in normal neutrophil counts for 6 months prior to HCT. Two patients received pre-HCT Interleukin-1 receptor antagonist (anakinra), without amelioration of cytopenias or immune dysregulation. Seven patients received granulocyte colony stimulating factor (G-CSF) for neutropenia, with no response.

\section{Immunological and Vasculitis Phenotype Pre-HCT}

IgG levels were low in $12 / 27$ tested patients, $\operatorname{Ig}$ A levels were low in $13 / 26$, and IgM levels were low in $15 / 27$ tested patients. Recurrent infections were reported in 17 of the 30 patients, mostly viral infections (in 14/17). Herpesvirus infections predominated, with three patients suffering from recurrent herpes zoster, one having protracted CMV infection, one with severe chicken pox, one with recurrent cutaneous HSV-1, two with HHV6 viremia, and four with EBV viremia ( 2 transient, 1 chronic, and 1 in the context of lymphoproliferative disease). Warts $(n=4)$, and mollusca contagiosum $(n=4)$ were also reported. Immunoglobulin substitution treatment was administered to 15 of the 30 patients before HCT, and splenomegaly was reported in 23/30 patients. Fifteen of 30 patients had reported vasculitis prior to HCT (Table 1): 9 had livedo racemosa, 3 had polyarteritis nodosa (PAN) (P6 with livedo, P7 with ICH and livedo, P11 


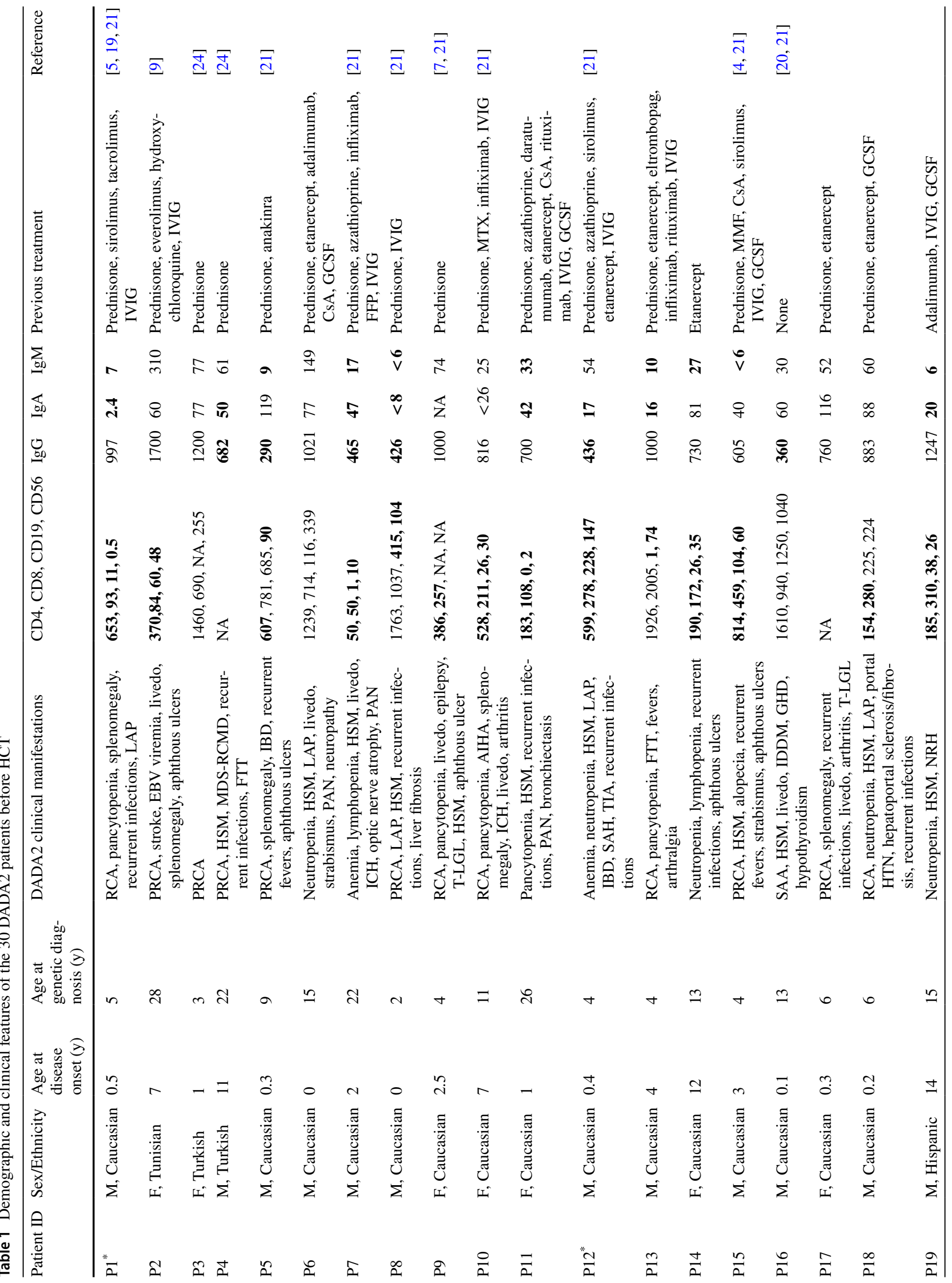




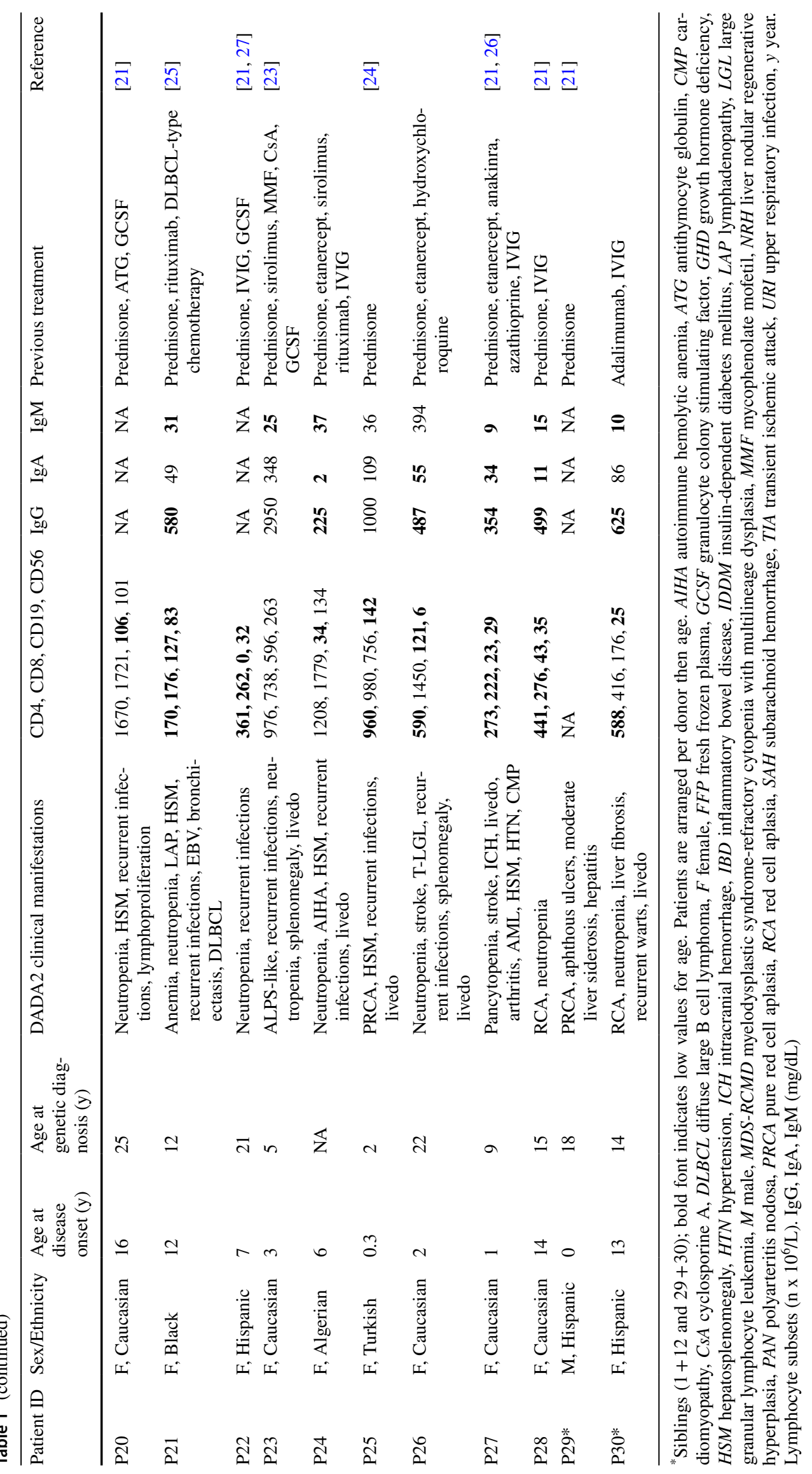


Table 2 Genetics and ADA2 enzymatic activity for the 30 DADA2 patients

\begin{tabular}{|c|c|c|c|c|}
\hline Patient ID & $A D A 2$ allele 1 & $A D A 2$ allele 2 & $\begin{array}{l}\text { ADA2 activity pre- } \\
\text { HCT }\end{array}$ & ADA2 activity post-HCT \\
\hline $\mathrm{P} 1^{*}$ & c.506G > A (p.R169Q) & c.506G > A (p.R169Q) & NA & $22.07^{\mathrm{a}}$ \\
\hline P2 & c. $\left(753+168 \_754-229\right)$ del & c. $\left(1081+139 \_1082-92\right)$ del & $2^{b}$ & $490^{\mathrm{b}}$ at $1 \mathrm{y}$ \\
\hline P3 & c.680-681del (p.Y227fs*27) & c.680-681del (p.Y227fs*27) & NA & NA \\
\hline P4 & c.1445 A > G (p.Y482C) & c.1445 A > G (p.Y482C) & NA & $44.38^{\mathrm{a}}$ \\
\hline P5 & c.144del (p.R49fs) & c. $47+2 \mathrm{~T}>\mathrm{C}$ (splice site $)$ & $0.2^{\mathrm{a}}$ & $11.7^{\mathrm{a}}$ \\
\hline P6 & c.506G > A (p.R169Q) & c.139G > T (p.G47W) & $0.37^{\mathrm{a}}$ & 1.67 of normal \\
\hline P7 & c.506C > T (p.R169Q) & c. $2 \mathrm{~T}>\mathrm{C}(\mathrm{p} . \mathrm{M} 1 \mathrm{~T})$ & NA & NA \\
\hline P8 & c.144delG (p.R49fs) & c.506G > A (p.R169Q) & NA & NA \\
\hline P9 & c.506G > A (p.R169Q) & c.506G > A (p.R169Q) & $0.0^{\mathrm{a}}$ & NA \\
\hline $\mathrm{P} 10$ & c.660C > A (p.Y220X) & c.660C > A (p.Y220X) & $2.5^{\mathrm{b}}$ & $403.6^{\mathrm{b}}$ at $2 \mathrm{y}$ \\
\hline P11 & c.3936delG (p.R131Sfs) & c.3936delG (p.R131Sfs) & $1.2^{\mathrm{b}}$ & NA \\
\hline $\mathrm{P} 12^{*}$ & c.506G > A (p.R169Q) & c.506G > A (p.R169Q) & $0.11^{\mathrm{a}}$ & $76.5^{\mathrm{b}}$ \\
\hline P13 & c.506G > A (p.R169Q) & c.506G > A (p.R169Q) & $0^{\mathrm{b}}$ & $77.8^{\mathrm{b}}$ at $2.5 \mathrm{~m}$ \\
\hline P14 & c.506G > A (p.R169Q) & c.506G > A (p.R169Q) & $0.09^{\mathrm{a}}$ & NA \\
\hline P15 & c.1110C > A (p.N370K) & c.1072G > A (p.G358R) & $0.6^{\mathrm{a}}$ & $19.7^{\mathrm{a}}$ at $1 \mathrm{y}$ \\
\hline P16 & c.506G > A (p.R169Q) & c.506G > A (p.R169Q) & NA & $8.3^{\mathrm{a}}$ at $10 \mathrm{y}$ \\
\hline P17 & c.506G > A (p.R169Q) & c.932 T > G (p.L311R) & $0.3^{\mathrm{a}}$ & NA \\
\hline $\mathrm{P} 18$ & c.336C > G (p.H112Q) & del exon 7 & $0.4^{\mathrm{a}}$ & 0.4 of normal \\
\hline P19 & c.506G > A (p.R169Q) & c.336C > G (p.H112Q) & NA & NA \\
\hline P20 & c.140G > T (p.G47V) & c.336C > G (p.H112Q) & NA & NA \\
\hline P21 & c.934C > T (p.R312X) & c.709delC (p.Glu237fs) & $0.2^{\mathrm{a}}$ & $35.6^{\mathrm{a}}$ at $2 \mathrm{~m}$ \\
\hline $\mathrm{P} 22$ & c. $794 C>$ G (p.S265X) & c.794C > G (p.S265X) & $0.0^{\mathrm{a}}$ & $10.8^{\mathrm{a}}$ at $1 \mathrm{y}$ \\
\hline $\mathrm{P} 23$ & c.1367A > G (p.Y456C) & c.1196. G > A (p.W399X) & NA & $21.4^{\mathrm{a}}$ \\
\hline P24 & c.140G > T (p.G47V) & c.140G > T (p.G47V) & NA & NA \\
\hline $\mathrm{P} 25$ & c. 1072 G > A (p.G358R) & c. $1072 \mathrm{G}>$ A (p.G358R) & $0.52^{\mathrm{a}}$ & NA \\
\hline P26 & p.Lys188Pro & g.17188016_17188596del & $0^{\mathrm{a}}$ & $6^{\mathrm{a}}$ \\
\hline P27 & c.506G > A (p.R169Q) & c.506G > A (p.R169Q) & $0.8^{\mathrm{a}}$ & $7.0^{\mathrm{a}}$ at $1 \mathrm{y}$ \\
\hline P28 & c.144dupG (p.R49fs) & c.506G > A (p.R169Q) & NA & NA \\
\hline P29* & c.506G > A (p.R169Q) & c. $1072 \mathrm{G}>\mathrm{A}(\mathrm{p} . \mathrm{G} 358 \mathrm{R})$ & NA & $22.3^{\mathrm{a}}$ \\
\hline $\mathrm{P} 30^{*}$ & c.506G > A (p.R169Q) & c. $1072 \mathrm{G}>\mathrm{A}(\mathrm{p} . \mathrm{G} 358 \mathrm{R})$ & $0.3^{\mathrm{a}}$ & NA \\
\hline
\end{tabular}

*Siblings $(1+12$ and $29+30)$

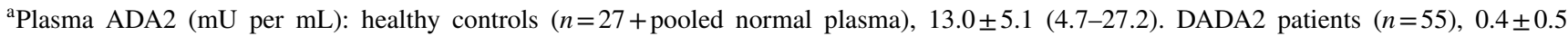
(0-2.5)

${ }^{\mathrm{b}}$ Dried plasma spots ADA2 (mU/g protein): healthy controls $(n=106), 130.0 \pm 53.2(24.9-285)$. DADA2 patients $(n=78), 4.7 \pm 4.8(0-23.3)$

isolated), three patients had ischemic stroke (P2, 26, 27), and four had intracranial hemorrhage (P7,10, 27, P12).

\section{Transplant Characteristics}

The indications for HCT were cytopenia with or without immunodeficiency and/or lymphoproliferation or malignancy (Table 3). The median age at HCT was 9 years (range: 2-28). Six of the 30 patients had received HCT before the description of DADA2 in 2014. Two patients (P16, P29) were inadvertently transplanted using affected siblings as donors and received salvage second HCT from unrelated donors. A total of 38 HCTs were performed for
30 patients. Twenty patients received MAC (P12 with two subsequent HCTs), eight received RIC, and two received NMA conditioning for the final curative transplant (Tables 3 and S1). The most commonly used regimen (in 11 patients) was treosulfan/fludarabine \pm thiotepa with antithymocyte globulin (ATG) or alemtuzumab. Serotherapy was used in 25/30 patients: ATG in 10 (rabbit ATG in all except horse ATG in P6 and P18) and alemtuzumab in 15 patients. The source of the stem cells for the final transplant was peripheral blood (PB) for 10 and bone marrow (BM) for 20 patients. Donors were HLA-matched related $(n=4)$, HLA-haploidentical sibling $(n=2), 10 / 10$ HLAmatched unrelated $(n=16)$, and 9/10 HLA-mismatched 


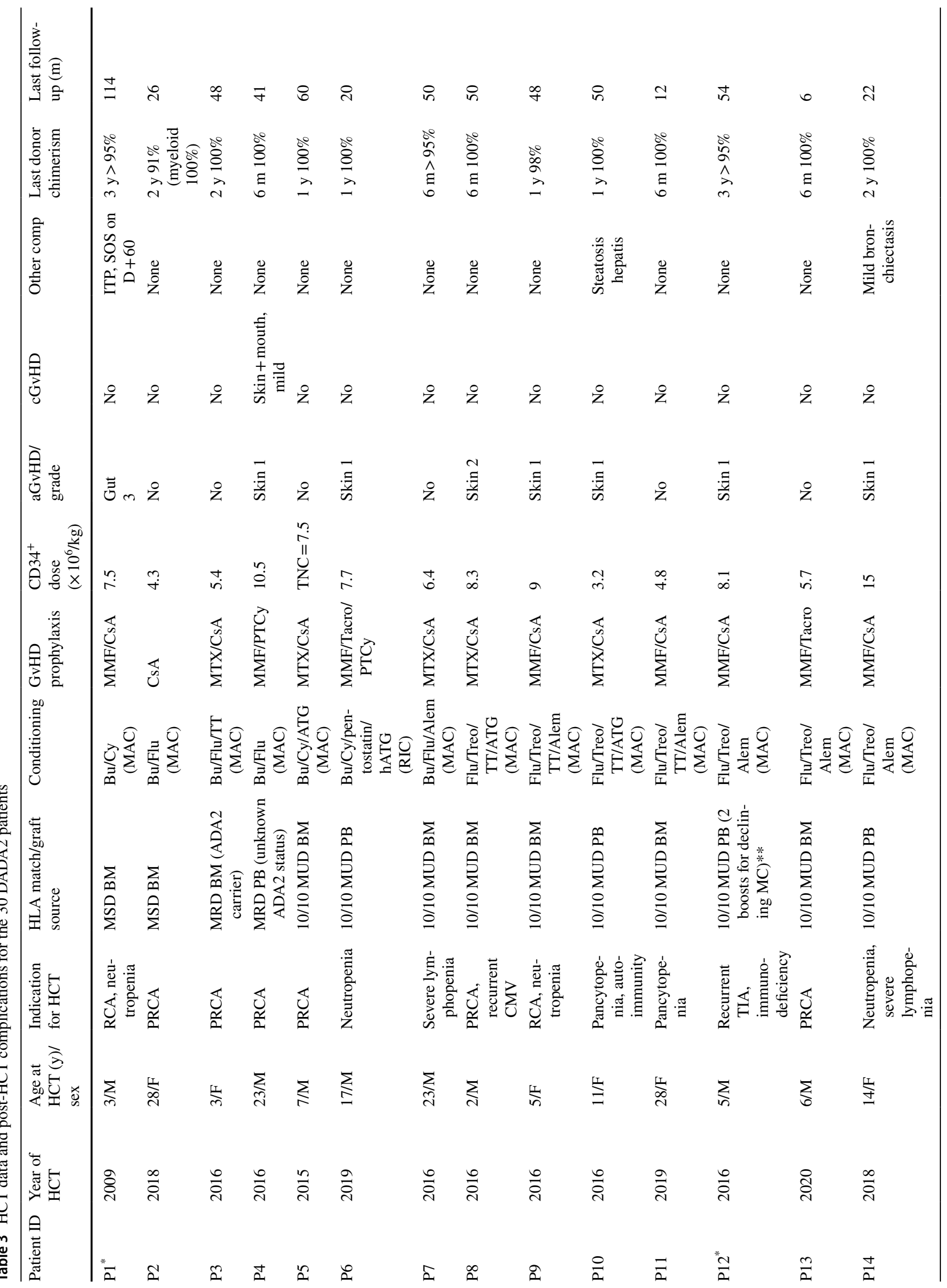




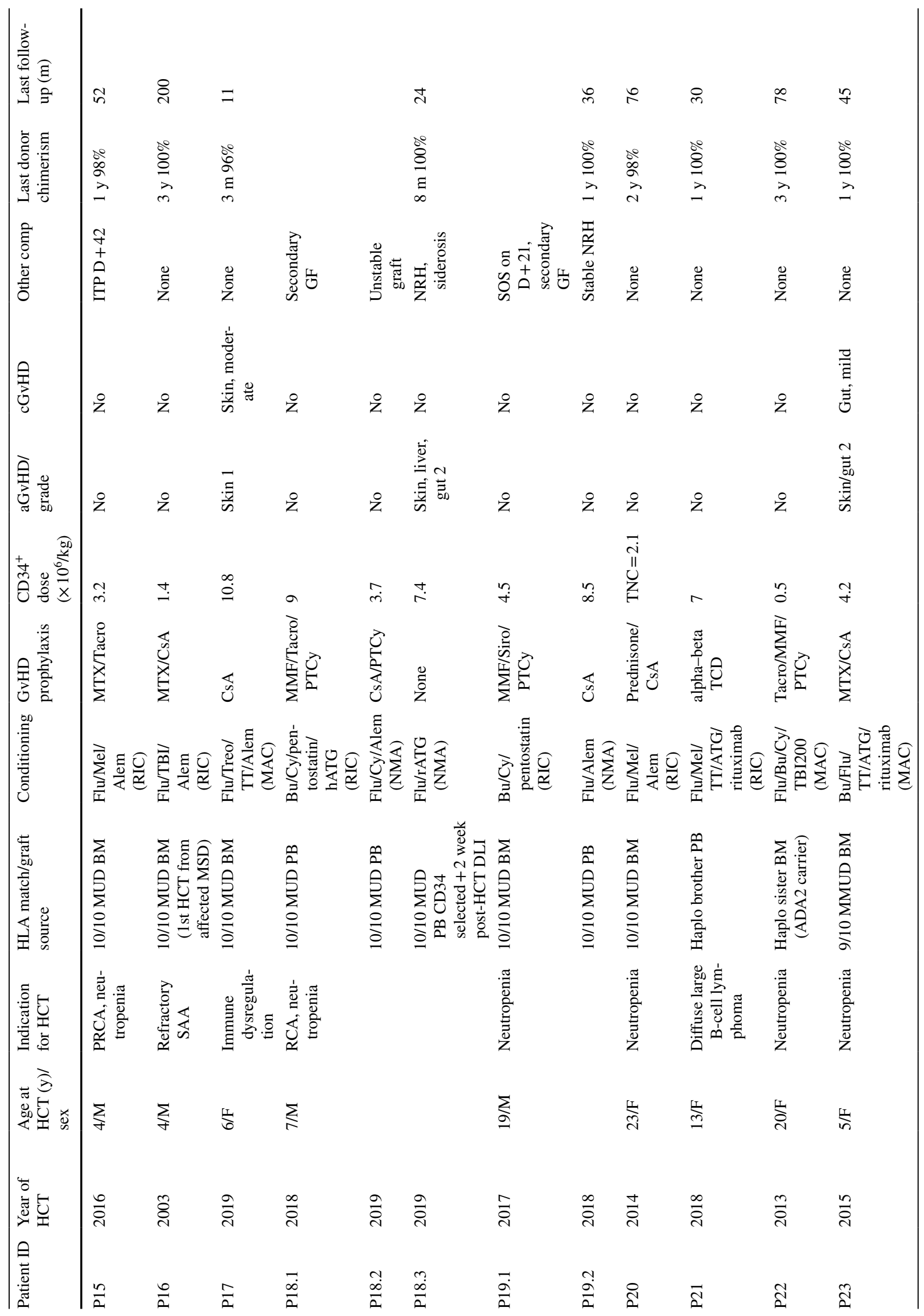




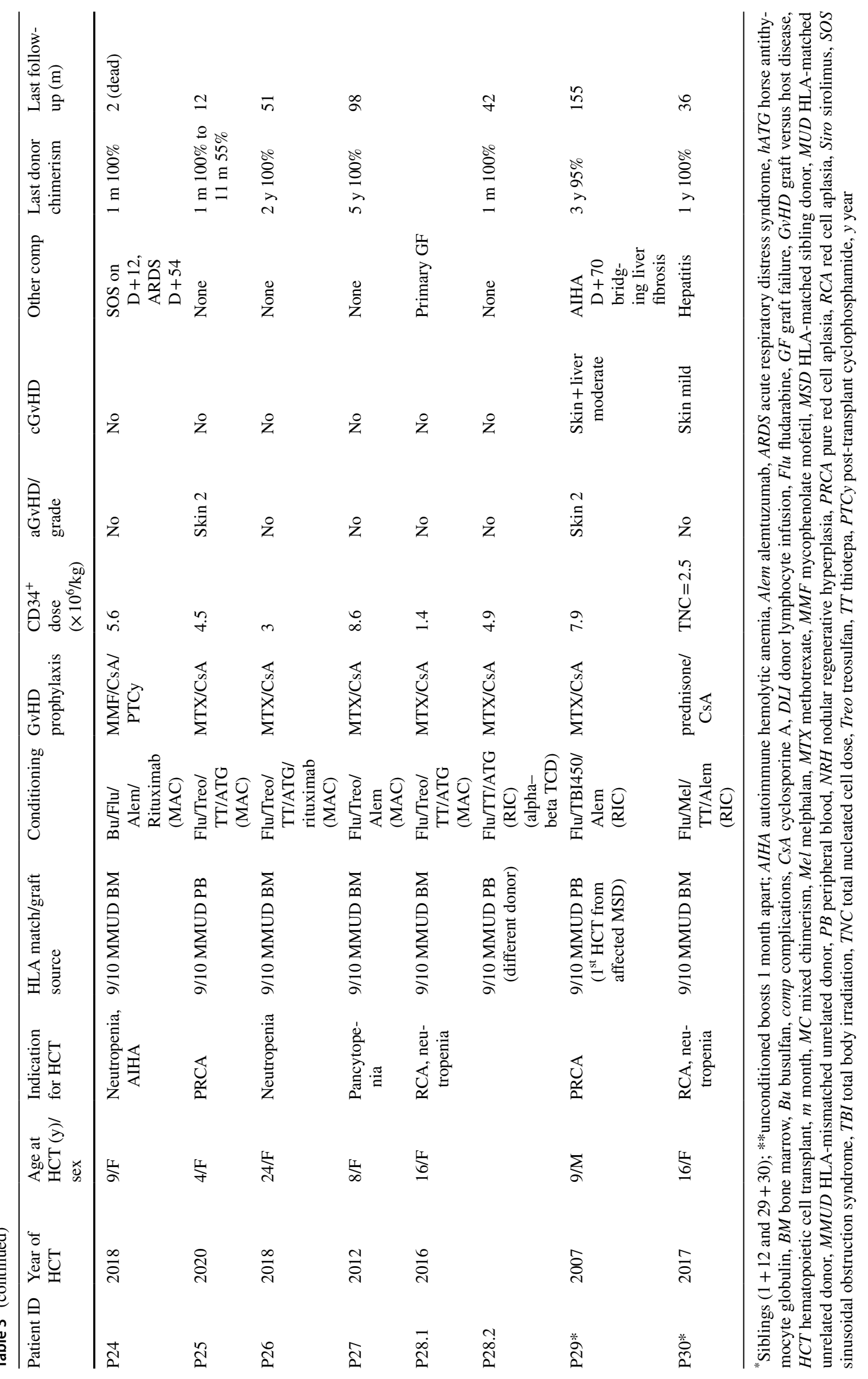


unrelated $(n=8)$ for the final transplant (Tables 3 and S1). Two of the six related donors carried heterozygous $A D A 2$ mutations (P3, P22), three were healthy (P1, P2, P21), and one donor was of unknown status (P4).

\section{Engraftment, Graft Failure, Transplant-Related Morbidity, Survival}

Median engraftment was $d+20$ for neutrophils and $d+23$ for platelets. In $25 / 28$ patients receiving grafts from unaffected donors, full donor chimerism was achieved by $\mathrm{d}+30$. Overall survival at 2 years was $97 \%$, with a median followup of survivors of 2 years (range: 0.5-16), accounting for 1545 patient-months post-HCT (Fig. 1A). P24 passed away 2 months post-HCT due to respiratory failure secondary to parainfluenza pneumonia despite full donor chimerism

A

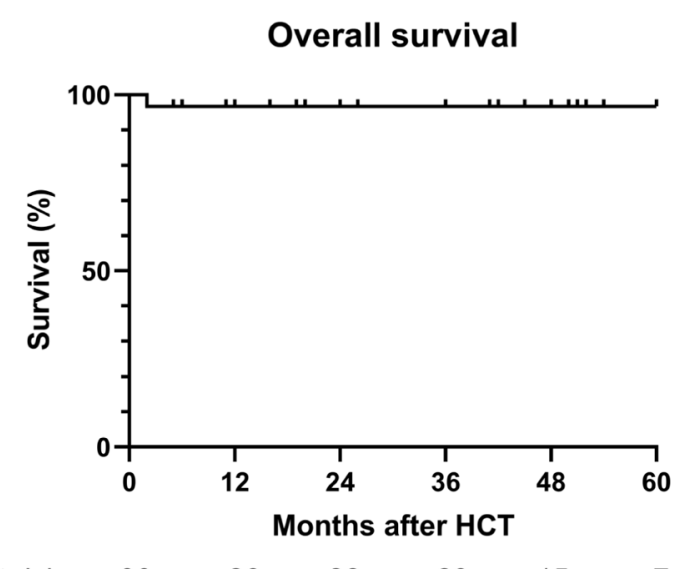

and treatment with steroids and etanercept for the suspicion of immune reconstitution inflammatory syndrome. Viral reactivation occurred in $17 / 30$ patients (56\%). Adenovirus, $\mathrm{CMV}$, and BK were most frequent involved, each observed in six patients. GRFS was $73 \%$ at 2 years, with all events occurring within the first year post-HCT. Of those transplanted with affected donors $(n=2), \mathrm{P} 16$ had primary graft failure requiring salvage HCT, and P29 had obtained nearfull donor chimerism but failed erythroid line engraftment and remained with PRCA. Three patients experienced graft failure. P18 required two and P19 one subsequent HCT for secondary GF. The latter two patients were found to have aggregates of $\mathrm{CD} 8+\mathrm{T}$ cells in their $\mathrm{BM}$ with low donor $\mathrm{T}$ cell chimerism (9\% and 0\%) (Fig. 1B, Fig. 2B). P28 required a second HCT likely due to low stem cell dose $\left(1.4 \times 10^{6} \%\right.$ $\mathrm{kg})$. P12 originally received MAC, but subsequently required

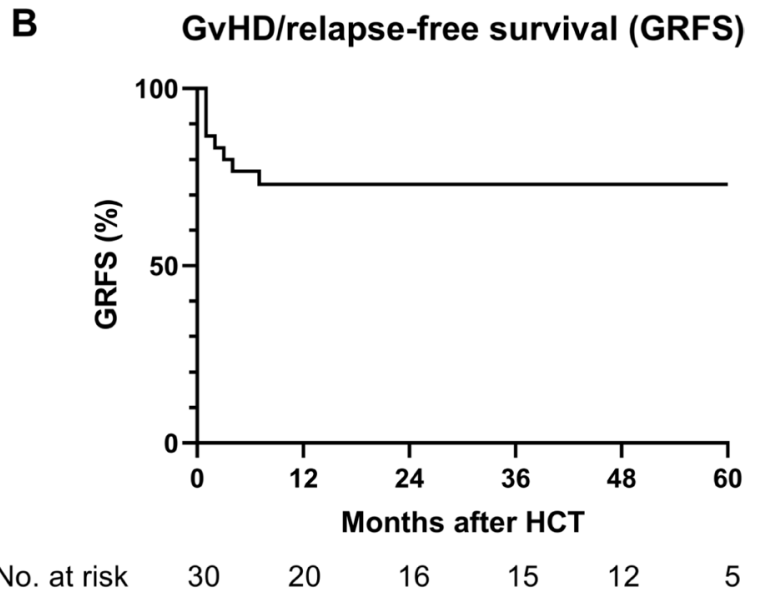

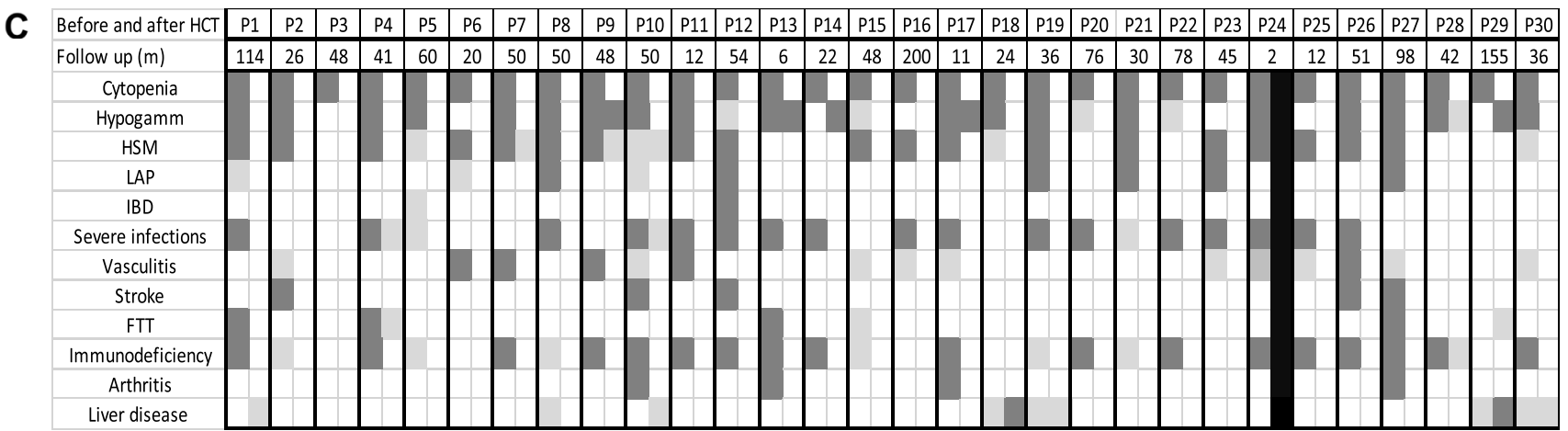

Fig. 1 Kaplan-Meier curves representing A overall survival, B GvHD-free, relapse-free survival (GRFS). GvHD relapse-free survival was calculated as the time from first HCT until the first occurrence of any of the following events: grades 3-4 aGvHD or moderate/ severe cGvHD GF, disease relapse (poor graft function/graft failure with DADA2 disease relapse requiring repeat transplant), or death. Overall survival is calculated on total number of patients $(n=30)$; GRFS is calculated on total number of HCT procedures, excluding the two procedures performed with stem cells from an affected sibling $(n=34)$. C Effect of HCT on clinical features resolution. Black squares indicate death post-HCT. Dark gray squares represent the presence of a clinical feature/phenotype. Light gray squares represent major improvement in clinical features. White squares represent complete resolution of clinical features. Each patient is presented by 2 attached columns (before and after HCT) for comparison. Followup time post-HCT for each patient is shown in months (second row). Severe infections represent any viral, bacterial, or fungal infection that required antiviral or antifungal treatment or led to sepsis. FTT, failure to thrive; HSM, hepatosplenomegaly; LAP, lymphadenopathy 
A

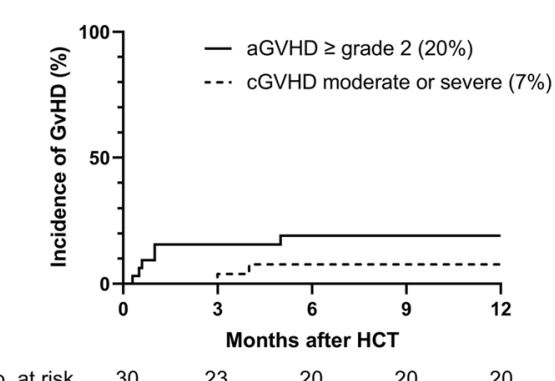

Fig. 2 Kaplan-Meier curves representing A cumulative incidence of GvHD (a, acute grade 2 or higher; c, chronic moderate or severe); B cumulative incidence of graft failure. Cumulative incidence of GvHD

two unconditioned HCTs due to drop in whole blood donor chimerism to $30 \%$ and new-onset RCA and agranulocytosis unresponsive to G-CSF. Cumulative incidence of aGvHD grades 2-4 was $20 \%$ at 1 year. Moderate-severe chronic GvHD developed in 2/30 patients (7\%) at 1 year (Fig. 2A). P1, P19, and P24 developed sinusoidal obstruction syndrome (SOS), which responded to fluid restriction and diuresis in P1 and P19 and to defibrotide in P24. All three patients with SOS received either high-dose cyclophosphamide or myeloablative busulfan.

\section{Cure of DADA2}

HCT cured the hematological phenotype in all patients, as confirmed at the most recent follow-up visit (Fig. 1C). No central vascular events were reported after engraftment (Fig. 1C). ADA2 plasma enzyme activity normalized in $16 / 17$ patients tested post-HCT (Table 2). This normalization occurred as early as $d+12$, coinciding with the reappearance of monocytes in the $\mathrm{PB}$, as demonstrated by the prospective monitoring of plasma ADA2 enzyme activity in one patient [29]. At last follow-up, 29 patients were still alive, and 28 of these patients displayed full donor chimerism. Donor chimerism fell to $55 \%$ in P25 but remained stable over several months, with no evidence of disease. Transient hematological autoimmunity post-HCT was reported in three patients: ITP in two and AIHA in one. This autoimmunity responded to various treatment regimens (intravenous immunoglobulins (IVIG)/steroids/sirolimus/rituximab/ bortezomib/romiplostim). Five patients are still on IVIG (one less than a year after HCT, one for mild bronchiectasis, and three post-rituximab treatment).
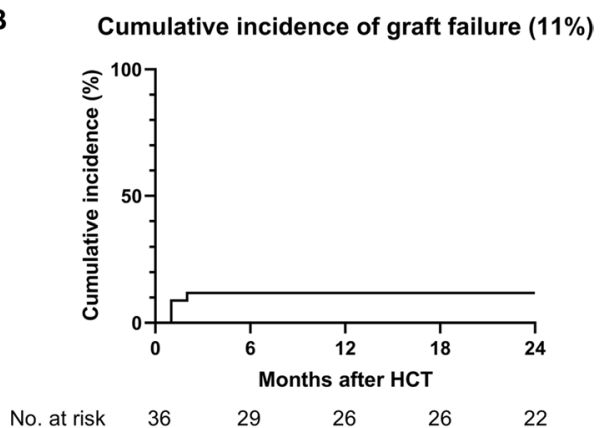

and cumulative incidence of graft failure are calculated on total number of HCT procedures, excluding the two procedures performed with stem cells from an affected sibling $(n=34)$

\section{Discussion}

We show here that HCT for DADA2 cures the hematological and immunological phenotypes of DADA2, with no new vascular events, with excellent survival, after a median follow-up of 2 years. Outside this study, two additional patients with DADA2 have received HCT and are reported to be alive and well [30, 31]. Another two patients have also undergone $\mathrm{HCT}$, one of whom died after receiving a graft from a donor heterozygous for the pathogenic mutation in ADA2 (P. Stepensky, personal communication). Adding these patients to the current report, 32 of the 34 DADA2 patients who have undergone HCT were cured. Despite the temporary resolution of neutropenia by a multidrug approach (cyclosporine, steroids, adalimumab) in one patient prior to HCT, this option is not feasible in the long term, and HCT is, therefore, a valuable alternative. HCT also resolved the vascular phenotype in all 15 patients with vasculitis. No additional central nervous system vascular events were reported postengraftment. Overall, the available data show an absence of new vascular events after engraftment post-HCT, demonstrating that hematopoietic cell-derived ADA2 plays a nonredundant role in restoring monocyte-endothelium interactions [12].

In the presence of this vascular phenotype, despite the impossibility of formal comparisons with other transplant indications, it is advisable to avoid high dose or untargeted busulfan and/or cyclophosphamide or high-dose radiation and to consider preventive measures for SOS. Liver disease was prominent and had a multifactorial etiology, directly related to DADA2 in some cases, but not in others (vasculitis, SOS, inflammation (DADA2/GvHD), iron overload, drug toxicity, GvHD). We therefore advise involving hepatologists in the care of DADA2 patients' right from diagnosis, with imaging, functional assessment, liver biopsy, and iron chelation if indicated, and opting for the least hepatotoxic conditioning regimen. The evaluation of pre/post-HCT 
renal disease, although less well described, requires a similarly high level of attention.

Three patients who were grafted with a non-diseased donor, suffered GF. Low CD $34^{+}$stem cell dose probably contributed to GF of P28. The choice of conditioning may have contributed to secondary GF in P18 and P19, both of whom had low donor $\mathrm{T}$ cell chimerism preceding GF. Our data thus suggest that robust host lymphodepletion, more than myeloablation, is essential in preventing GF. It remains a matter of debate whether a related donor with a single deleterious allele of $A D A 2$ can be considered a suitable donor. Two of the six related donors carried heterozygous $A D A 2$ mutations (P3, P22: both HCT were successful), three were healthy (P1, P2, P21: all HCTs were successful), and one donor was of unknown status (P4). P25 has suffered no disease relapse, with a whole blood donor chimerism of 55\%. In P12, whole blood donor chimerism fell to $30 \%$, resulting in disease relapse, suggesting that there is a minimum required level of donor chimerism in DADA2. Indeed, patients carrying only a single pathogenic allele, with intermediate levels of ADA2 enzyme activity, have been reported to manifest DADA2 symptoms [10, 32]. In contrast, 9/10 MMUD and MUD may be considered suitable options for donors: There were 16 MUD and 8 MMUD transplants in this cohort.

The indication for HCT in this cohort was cytopenia and/ or malignancy and immunodeficiency, not responding to treatment with TNF inhibitors. DADA2 patients with refractory BMF or immune cytopenia should be referred early on for HCT evaluation, given the morbidity and mortality due to hemorrhage, iron overload, infection, and long-term treatment with multiple immunosuppressive agents [18]. HCT is also a treatment option for patients who do not have access to anti-TNF agents even in the absence of immune cytopenia, BMF, or immunodeficiency. Theoretically, HCT could be a treatment option for patients on long-term treatment with anti-TNF inhibitors who develop neutralizing anti-drug antibodies [33]. Gene therapy (GT) is a promising option for the future, but is unlikely to be available to all DADA2 patients worldwide. In addition, GT requires conditioning and the presence of a sufficient number of autologous hematopoietic stem cells and may fail to reverse immune cytopenias if residual host $\mathrm{T}$ cells remain. Thus, GT is only feasible in patients without refractory cytopenia/BMF/malignancy, without prohibitive organ dysfunction, and without host $\mathrm{T}$ cell-mediated cytopenias. In the latter, HCT is likely the only potential curative treatment. For both HCT and GT, it emerges that full replacement of the host $\mathrm{T}$ cell compartment, and at least partial replacement of the myeloid compartment with ADA2 sufficient cells, is required.

In conclusion, we report here experience with the treatment of 30 DADA2 patients with cytopenia, BMF and immunodeficiency by HCT. All but one of the patients are alive and well and are cured at a median follow-up of
2 years. This successful treatment of an auto-inflammatory condition paves the way for application of HCT in other auto-inflammatory conditions refractory to classic immunosuppressive approaches.

Supplementary Information The online version contains supplementary material available at https://doi.org/10.1007/s10875-021-01098-0.

Acknowledgements We thank the affected children and their parents for their participation and their confidence. We thank the DADA2 Foundation (www.dada2.org) for organizing the Inaugural International Conference on the Deficiency of ADA2, with special thanks to Chip Chambers, MD for forming the foundation. We thank Ms. Jo Vencken for technical assistance and all colleagues who contributed to the care for these patients. We thank Nancy J. Ganson, PhD and Susan J. Kelly, PhD at Duke University Center, Durham, for performing numerous ADA2 enzyme activity measurements. We also thank Amy Cruickshank, DO at the University of New Mexico, for her contributions. This study was made possible by collaboration with the European Society of Blood and Bone Marrow Transplantation (EBMT), Inborn Errors Working Party (IEWP), and the DADA2 Foundation and European Society for Immunodeficiencies. SU and NA would like to thank ERARE for supporting DBA molecular work-up as a part of the EuroDBA Project. This work is supported by ERN-RITA.

Author Contribution HH, GB, and I. Meyts collected, analyzed, and interpreted data and wrote the manuscript. HH, SO, SU, IOB, NA, MT, MK, JS, DD, DDH, APH, SMH, RK, GS, ARK, I. Müller, MdS, SD, F. Babor, F. Barzaghi, RB, JVM, VB, SC, MPC, PS, NB, DM, GLG, DB, J. Dalal, JB, ED, J. Dara, CLL, SH, SJ, YK, TG, LM, CS, FC, SS, AS, $\mathrm{KW}, \mathrm{CC}, \mathrm{MH}, \mathrm{AO}, \mathrm{JK}$, and I. Meyts provided clinical information from patients and edited the manuscript. MH provided the ADA2 enzyme assay activity data for most of the patients and edited the manuscript. GB helped with data analysis. All the authors contributed to clinical care, data gathering and analysis and edited and approved the final manuscript.

Funding This project was funded in whole or in part by federal funds from the National Cancer Institute, National Institutes of Health, under Contract No. HHSN261200800001E. This research was supported (in part) by the Intramural Research Program of the NIH, National Cancer Institute, Center for Cancer Research and (in part) by the National Institute of Allergy and Infectious Diseases, the Sir Jules Thorn Charitable Trust (12/JTA), and the Wellcome Trust (207556_Z_17_Z). Specific ERARE grant funding was attributed to the EuroDBA researchers of Turkey by the Scientific and Technological Research Council of Turkey (TÜBITAK, 315S192).SD is supported by the Personal Research Foundation - Flanders grant 11F4421N. CLL received support from the Immune Deficiency Foundation and Yale University School of Medicine. IM is a Senior Clinical Investigator at the Research Foundation - Flanders and is supported by the CSL Behring Chair of Primary Immunodeficiencies paid to KU Leuven; by the KU Leuven C1 Grant C16/18/007; by a VIB GC PID Grant, by the FWO Grants G0C8517N, G0B5120N, and G0E8420N; and by the Jeffrey Modell Foundation. This project has received funding from the European Research Council (ERC) under the European Union's Horizon 2020 research and innovation program (grant agreement No. 948959, MORE2ADA2).

Availability of Data and Materials Available upon request to the corresponding authors. 


\section{Declarations}

Ethics Approval This retrospective study was approved by the Ethics Committee of Leuven University Hospitals (study number S63982 and S63077) and by individual local Ethics Committees.

Consent to Participate Written informed consent was obtained from participants or their guardians.

Consent for Publication Patients signed informed consent regarding publishing their data.

Conflict of Interest The authors have no competing financial interests to declare with respect to this work. IM is holder of CSL-Behring Chair paid to KU Leuven. ARK is a speaker for SOBI. JVM served on an advisory Board for Takeda.

Disclaimer The content of this publication does not necessarily reflect the views or policies of the Department of Health and Human Services, nor does the mention of trade names, commercial products, or organizations imply endorsement by the US government.

Open Access This article is licensed under a Creative Commons Attribution 4.0 International License, which permits use, sharing, adaptation, distribution and reproduction in any medium or format, as long as you give appropriate credit to the original author(s) and the source, provide a link to the Creative Commons licence, and indicate if changes were made. The images or other third party material in this article are included in the article's Creative Commons licence, unless indicated otherwise in a credit line to the material. If material is not included in the article's Creative Commons licence and your intended use is not permitted by statutory regulation or exceeds the permitted use, you will need to obtain permission directly from the copyright holder. To view a copy of this licence, visit http://creativecommons.org/licenses/by/4.0/.

\section{References}

1. Zhou Q, Yang D, Ombrello AK, Zavialov AV, Toro C, Zavialov $\mathrm{AV}$, et al. Early-onset stroke and vasculopathy associated with mutations in ADA2. N Engl J Med. 2014;370(10):911-20.

2. NavonElkan P, Pierce SB, Segel R, Walsh T, Barash J, Padeh S, et al. Mutant adenosine deaminase 2 in a polyarteritis nodosa vasculopathy. N Engl J Med. 2014;370(10):921-31.

3. Caorsi R, Penco F, Schena F, Gattorno M. Monogenic polyarteritis: the lesson of ADA2 deficiency. Pediatr Rheumatol Online J. 2016;14(1):51.

4. Hashem H, Egler R, Dalal J. Refractory pure red cell aplasia manifesting as deficiency of adenosine deaminase 2. J Pediatr Hematol Oncol. 2017;39(5):e293-6.

5. Van Eyck Jr L, Hershfield MS, Pombal D, Kelly SJ, Ganson NJ, Moens L, et al. Hematopoietic stem cell transplantation rescues the immunologic phenotype and prevents vasculopathy in patients with adenosine deaminase 2 deficiency. J Allergy Clin Immunol. 2015;135(1):283-7 e5.

6. Ben-Ami T, Revel-Vilk S, Brooks R, Shaag A, Hershfield MS, Kelly SJ, et al. Extending the clinical phenotype of adenosine deaminase 2 deficiency. J Pediatr. 2016;177:316-20.

7. Trotta L, Martelius T, Siitonen T, Hautala T, Hamalainen S, Juntti $\mathrm{H}$, et al. ADA2 deficiency: clonal lymphoproliferation in a subset of patients. J Allergy Clin Immunol. 2018;141(4):1534-7.
8. Alabbas F, Elyamany G, Alsharif O, Hershfield M, Meyts I. Childhood Hodgkin lymphoma: think DADA2. J Clin Immunol. 2019;39(1):26-9.

9. Le Voyer T, Boutboul D, Ledoux-Pilon A, de Fontbrune FS, Boursier G, Latour S, et al. Late-onset EBV susceptibility and refractory pure red cell aplasia revealing DADA2. J Clin Immunol. 2020;40(6):948-53.

10. Schnappauf O, Zhou Q, Moura NS, Ombrello AK, Michael DG, Deuitch N, et al. Deficiency of adenosine deaminase 2 (DADA2): hidden variants, reduced penetrance, and unusual inheritance. J Clin Immunol. 2020;40(6):917-26.

11. Martinon F, Aksentijevich I. New players driving inflammation in monogenic autoinflammatory diseases. Nat Rev Rheumatol. 2015;11(1):11-20.

12. Dhanwani R, Takahashi M, Mathews IT, Lenzi C, Romanov A, Watrous JD, et al. Cellular sensing of extracellular purine nucleosides triggers an innate IFN-beta response. Sci Adv. 2020;6(30):eaba3688.

13. Meyts I, Aksentijevich I. Deficiency of adenosine deaminase 2 (DADA2): updates on the phenotype, genetics, pathogenesis, and treatment. J Clin Immunol. 2018;38(5):569-78.

14. Sahin S, Adrovic A, Kasapcopur O. A monogenic autoinflammatory disease with fatal vasculitis: deficiency of adenosine deaminase 2. Curr Opin Rheumatol. 2020;32(1):3-14.

15. Sharma A, Naidu G, Sharma V, Jha S, Dhooria A, Dhir V, et al. Deficiency of adenosine deaminase 2 (DADA2) in adults and children: experience from India. Arthritis Rheumatol. 2020;73(2):276-285

16. Ombrello AK, Qin J, Hoffmann PM, Kumar P, Stone D, Jones A, et al. Treatment strategies for deficiency of adenosine deaminase 2 N Engl J Med. 2019;380(16):1582-4.

17. Cooray S, Omyinmi E, Hong Y, Papadopoulou C, Harper L, Al-Abadi E, et al. Anti-tumour necrosis factor treatment for the prevention of ischaemic events in patients with deficiency of adenosine deaminase 2 (DADA2). Rheumatology (Oxford). 2021. https://doi.org/10.1093/rheumatology/keaa837

18. Lee PY, Kellner ES, Huang Y, Furutani E, Huang Z, Bainter W, et al. Genotype and functional correlates of disease phenotype in deficiency of adenosine deaminase 2 (DADA2). J Allergy Clin Immunol. 2020;145(6):1664-72 e10.

19. Van Eyck L, Liston A, Wouters C. Mutant ADA2 in vasculopathies. N Engl J Med. 2014;371(5):480.

20. van Montfrans J, Zavialov A, Zhou Q. Mutant ADA2 in vasculopathies. N Engl J Med. 2014;371(5):478.

21. Hashem H, Kumar AR, Muller I, Babor F, Bredius R, Dalal J, et al. Hematopoietic stem cell transplantation rescues the hematological, immunological, and vascular phenotype in DADA2. Blood. 2017;130(24):2682-8.

22. Lee SJ. Classification systems for chronic graft-versus-host disease. Blood. 2017;129(1):30-7.

23. Barzaghi F, Minniti F, Mauro M, Bortoli M, Balter R, Bonetti E, et al. ALPS-Like phenotype caused by ADA2 deficiency rescued by allogeneic hematopoietic stem cell transplantation. Front Immunol. 2018;9:2767.

24. Ozen S, Batu ED, Taskiran EZ, Ozkara HA, Unal S, Guleray $\mathrm{N}$, et al. A Monogenic disease with a variety of phenotypes: deficiency of adenosine deaminase 2. J Rheumatol. 2020;47(1):117-25.

25. Brooks JP, Rice AJ, Ji W, Lanahan SM, Konstantino M, Dara $\mathrm{J}$, et al. Uncontrolled Epstein-Barr virus as an atypical presentation of deficiency in ADA2 (DADA2). J Clin Immunol. 2021;41(3):680-3.

26. Van Montfrans JM, Hartman EA, Braun KP, Hennekam EA, Hak EA, Nederkoorn PJ, et al. Phenotypic variability in patients with ADA2 deficiency due to identical homozygous R169Q mutations. Rheumatology (Oxford). 2016;55(5):902-10. 
27. Hsu AP, West RR, Calvo KR, Cuellar-Rodriguez J, Parta M, Kelly SJ, et al. Adenosine deaminase type 2 deficiency masquerading as GATA2 deficiency: successful hematopoietic stem cell transplantation. J Allergy Clin Immunol. 2016;138(2):628-30 e2.

28. Itan Y, Bryson K, Thomas MG. Detecting gene duplications in the human lineage. Ann Hum Genet. 2010;74(6):555-65.

29. Bucciol G, Delafontaine S, Segers H, Bossuyt X, Hershfield MS, Moens L, et al. Hematopoietic stem cell transplantation in ADA2 deficiency: early restoration of ADA2 enzyme activity and disease relapse upon drop of donor chimerism. J Clin Immunol. 2017;37(8):746-50.

30. Gibson PG. Allergic bronchopulmonary aspergillosis. Semin Respir Crit Care Med. 2006;27(2):185-91.

31. Liu L, Wang W, Wang Y, Hou J, Ying W, Hui X, et al. A Chinese DADA2 patient: report of two novel mutations and successful HSCT. Immunogenetics. 2019;71(4):299-305.
32. Carmona-Rivera C, Khaznadar SS, Shwin KW, Irizarry-Caro JA, O'Neil LJ, Liu Y, et al. Deficiency of adenosine deaminase 2 triggers adenosine-mediated NETosis and TNF production in patients with DADA2. Blood. 2019;134(4):395-406.

33. Klareskog L, Gaubitz M, Rodriguez-Valverde V, Malaise M, Dougados M, Wajdula J, et al. Assessment of long-term safety and efficacy of etanercept in a 5-year extension study in patients with rheumatoid arthritis. Clin Exp Rheumatol. 2011;29(2):238-47.

Publisher's Note Springer Nature remains neutral with regard to jurisdictional claims in published maps and institutional affiliations.

\section{Authors and Affiliations}

Hasan Hashem ${ }^{1} \cdot$ Giorgia Bucciol $^{2,3} \cdot$ Seza Ozen $^{4,5} \cdot$ Sule Unal ${ }^{6} \cdot$ lkbal Ok Bozkaya $^{7}$. Nurten Akarsu ${ }^{8}$. Mervi Taskinen $^{9} \cdot$ Minna Koskenvuo $^{10}$. Janna Saarela ${ }^{11,12}$. Dimana Dimitrova ${ }^{13}$. Dennis D. Hickstein ${ }^{14}$. Amy P. Hsu ${ }^{15}$. Steven M. Holland ${ }^{15} \cdot$ Robert Krance $^{16}$. Ghadir Sasa ${ }^{16}$. Ashish R. Kumar ${ }^{17,18} \cdot$ Ingo Müller $^{19}$. Monica Abreu de Sousa ${ }^{19}$. Selket Delafontaine ${ }^{2,3}$. Leen Moens ${ }^{3}$. Florian Babor ${ }^{20}$. Federica Barzaghi ${ }^{21}$. Maria Pia Cicalese ${ }^{22}$. Robbert Bredius ${ }^{23}$. Joris van Montfrans ${ }^{24}$. Valentina Baretta ${ }^{25}$. Simone Cesaro ${ }^{25}$. Polina Stepensky ${ }^{26}$. Neven Benedicte ${ }^{27}$. Despina Moshous ${ }^{27}$. Guillaume Le Guenno ${ }^{28}$. David Boutboul ${ }^{29}$. Jignesh Dalal ${ }^{30}$. Joel P. Brooks ${ }^{31}$. Elif Dokmeci' ${ }^{32}$. Jasmeen Dara ${ }^{33}$. Carrie L. Lucas ${ }^{31}$. Sophie Hambleton ${ }^{34}$. Keith Wilson ${ }^{35}$. Stephen Jolles ${ }^{36}$. Yener Koc ${ }^{37}$ - Tayfun Güngör ${ }^{38}$. Caroline Schnider ${ }^{39}$. Fabio Candotti ${ }^{40}$. Sandra Steinmann ${ }^{41} \cdot$ Ansgar Schulz $^{41}$. Chip Chambers ${ }^{42} \cdot$ Michael Hershfield $^{43} \cdot$ Amanda Ombrello $^{44}$. Jennifer A. Kanakry ${ }^{13} \cdot$ Isabelle Meyts ${ }^{2,3}$

1 Department of Pediatrics, Division of Pediatric Hematology and Oncology, Bone Marrow Transplant Unit, King Hussein Cancer Center (KHCC), P.O Box 1269, Amman 11941, Jordan

2 Department of Pediatrics, ERN RITA Core Center, University Hospitals Leuven, Herestraat 49, 3000 Leuven, Belgium

3 Department of Microbiology, Immunology and Transplantation, Laboratory for Inborn Errors of Immunity, University Hospitals Leuven, Herestraat 49, 3000 Leuven, Belgium

4 Department of Pediatric Rheumatology, Hacettepe University, Ankara, Turkey

5 Hacettepe University Vasculitis Research Center, Ankara, Turkey

6 Department of Pediatric Hematology, Research Center for Fanconi Anemia and Other Inherited Bone Marrow Failure Syndromes, Hacettepe University, Ankara, Turkey

7 Division of Pediatric Hematology and Oncology, Bone Marrow Transplant Unit, University of Health Sciences, Ankara City Hospital, Ankara, Turkey

8 Department of Medical Genetics, Hacettepe University, Sihhiye, 06100 Ankara, Turkey

9 Division of Pediatric Hematology, Oncology and Stem Cell Transplantation, Helsinki University Hospital, Helsinki, Finland
10 Pediatric Hematology, Oncology and Stem Cell Transplantation, Children and Adolescents, Helsinki University Hospital, Helsinki, Finland

11 Institute for Molecular Medicine Finland, HiLIFE, University of Helsinki, Helsinki, Finland

12 Centre for Molecular Medicine Norway, University of Oslo, Oslo, Norway

13 Experimental Transplantation and Immunotherapy Branch, National Cancer Institute of the National Institutes of Health, Bethesda, MD, USA

14 Immune Deficiency Cellular Therapy Program, CCR, NCI, MD, Bethesda, USA

15 Laboratory of Clinical Infectious Diseases, National Institute of Allergy and Infectious Diseases, Bethesda, MD, USA

16 Cell and Gene Therapy, Baylor College of Medicine, Houston, TX, USA

17 Cincinnati Children's Hospital Medical Center, Cincinnati, $\mathrm{OH}, \mathrm{USA}$

18 University of Cincinnati College of Medicine, Cincinnati, $\mathrm{OH}$, USA

19 Division of Pediatric Stem Cell Transplantation and Immunology, University Medical Center Hamburg-Eppendorf, Hamburg, Germany

20 Department of Pediatric Oncology, Hematology and Clinical Immunology, Center for Child and Adolescent Health, 
Medical Faculty, Heinrich-Heine-University, Düsseldorf, Germany

21 San Raffaele Telethon Institute for Gene Therapy (TIGET), Pediatric Immunohematology and Bone Marrow Transplantation Unit, IRCCS San Raffaele Scientific Institute Milan, Milan, Italy

22 Pediatric Immunohematology and Bone Marrow Transplantation Unit, IRCCS San Raffaele Scientific Institute, Milan, Italy

23 Department of Pediatrics, Willem-Alexander Children's Hospital, Leiden University Medical Center, Leiden, Netherlands

24 Department of Pediatric Immunology and Infectious Diseases, Wilhelmina Children's Hospital, University Medical Centre Utrecht, Utrecht, Netherlands

25 Pediatric Hematology Oncology, Department of Mother and Child, Azienda Ospedaliera Universitaria Integrata, Verona, Italy

26 Department of Bone Marrow Transplantation and Cancer Immunotherapy, Hadassah University Medical Center, Jerusalem, Israel

27 Pediatric Immunology, Hematology and Rheumatology Unit, Hôpital Necker-Enfants Malades, APHP, Paris, France

28 Department of Internal Medicine, University Hospital Estaing, CHU Clermont-Ferrand, Clermont-Ferrand, France

29 Clinical Immunology Department, Hospital Saint Louis, Université de Paris, Paris, France

30 Rainbow Babies and Children's Hospital, Case Western Reserve University, Cleveland, OH, USA

31 Department of Immunobiology, Yale University School of Medicine, New Haven, CT, USA

32 Department of Pediatrics, University of New Mexico, Albuquerque, NM, USA
33 Department of Pediatrics, Division of Allergy, Immunology, Blood and Marrow Transplantation, University of California San Francisco, San Francisco, CA, USA

34 Newcastle University Translational and Clinical Research Institute and Great North Children's Hospital, Newcastle Upon Tyne Hospitals NHS Foundation Trust, , Newcastle Upon Tyne, UK

35 Department of Hematology, University Hospital of Wales, Cardiff, UK

36 Immunodeficiency Centre for Wales, University Hospital of Wales, Cardiff, UK

37 Stem Cell Transplant Unit, Medicana International, Istanbul, Turkey

38 Division of Hematology/Oncology/Immunology, Gene Therapy, and Stem Cell Transplantation, University Children's Hospital Zurich - Eleonore Foundation \& Children's Research Center (CRC), Steinwiesstrasse 75, CH-8032 Zurich, Switzerland

39 Pediatric Immuno-Rheumatology of Western Switzerland, Department Women-Mother-Child, Lausanne University Hospital, Lausanne, Switzerland

40 Division of Immunology and Allergy, Lausanne University Hospital and University of Lausanne, Lausanne, Switzerland

41 Department of Pediatrics, University Medical Center Ulm, Ulm, Germany

42 Vanderbilt University Medical Center, Nashville, TN, USA

43

Department of Medicine and Biochemistry, Duke University Medical Center, Durham, NC, USA

44 Metabolic, Cardiovascular, and Inflammatory Disease Genomics Branch, National Human Genome Research Institute (NHGRI), Bethesda, MD, USA 